OPEN ACCESS

Edited by:

Jonatan Barrera-Chimal, National Autonomous University of Mexico, Mexico

Reviewed by:

Audrey Adji,

Victor Chang Cardiac Research Institute, Australia Gergo A. Molnar.

University of Pécs, Hungary

*Correspondence:

Jun Sung Moon mjs7912@yu.ac.kr

tThese authors have contributed equally to this work

Specialty section:

This article was submitted to Nephrology,

a section of the journa

Frontiers in Medicine

Received: 30 July 2020

Accepted: 24 August 2020

Published: 30 September 2020

Citation:

Lim TH, Chung SM, Lee DS, Choi SR, Moon JS, Yoon JS, Won KC and Lee HW (2020) Peripheral Arterial

Stiffness Increases the Risk of Progression of Renal Disease in Type

2 Diabetic Patients.

Front. Med. 7:588967.

doi: 10.3389/fmed.2020.588967

\section{Peripheral Arterial Stiffness Increases the Risk of Progression of Renal Disease in Type 2 Diabetic Patients}

\author{
Tae Hoon Lim ${ }^{1 t}$, Seung Min Chung ${ }^{2 \dagger}$, Dong Sung Lee ${ }^{1}$, Se Ra Choi ${ }^{1}$, Jun Sung Moon ${ }^{2 *}$, \\ Ji Sung Yoon ${ }^{2}$, Kyu Chang Won ${ }^{2}$ and Hyoung Woo Lee ${ }^{2}$
}

${ }^{1}$ College of Medicine, Yeungnam University, Daegu, South Korea, ${ }^{2}$ Division of Endocrinology and Metabolism, Department of Internal Medicine, Yeungnam University Medical Center, Daegu, South Korea

Aims: Our aim was to investigate the effects of peripheral arterial stiffness on the risk of progression of renal disease in patients with type 2 diabetes (T2D).

Methods: This was a single center, retrospective cohort study. Brachial-ankle pulse wave velocity (baPWV) tests were performed on T2D patients in 2015. Increased arterial stiffness was defined as baPWV of $\geq 1800 \mathrm{~cm} / \mathrm{s}$. We applied criteria for progression of renal disease according to EMPA-REG OUTCOME trial.

Results: In total, 186 patients were enrolled in the final study. The mean age was 59.1 years and male:female ratio was 1.73:1. Thirteen (7\%) patients progressed to renal disease during the average follow-up time of 35.3 months. In particular, the risk of progression to macroalbuminuria was significantly higher in the baPWV $\geq 1800$ $\mathrm{cm} / \mathrm{s}$ group (HR 6.216, $p=0.020$ ). Individuals with a baPWV of $\geq 1800 \mathrm{~cm} / \mathrm{s}$ (when comparisons were adjusted for age, sex, blood pressure, diabetes duration, eGFR, and use of renin-angiotensin system inhibitors) had a significantly higher risk of the progression of renal disease $(\mathrm{HR}=8.480, p=0.014)$.

Conclusion: These results suggest that peripheral arterial stiffness (baPWV $\geq 1800$ $\mathrm{cm} / \mathrm{s}$ ) may be a risk factor for the progression of renal disease in T2D patients.

Keywords: arterial stiffness, diabetes mellitus, diabetic nephropathy, pulse wave velocity, renal function decline

\section{INTRODUCTION}

Type 2 diabetes mellitus (T2D), along with hypertension, is one of the leading chronic diseases in Korea (1). In addition to the increasing prevalence of diabetes, diabetic complications such as cardiovascular disease, retinopathy, neuropathy, and nephropathy have significant negative effects on quality of life among T2D patients.

Aside from the microvascular complications that affect T2D patients, diabetic kidney disease (DKD) is the most common cause of end-stage renal disease (ESRD) resulting in the increased morbidity and mortality of diabetic patients. According to the Korean Society of Nephrology, for the past 20 years diabetes has been identified as the leading cause of ESRD requiring renal replacement treatment, accounting for $48.8 \%$ of cases (2). ESRD causes a heavy socioeconomic and health burden (3), so it is important to closely monitor T2D 
patients and try to preserve renal function, as the progression of renal disease is often irreversible (4). Recent guidelines strongly recommend the annual screening of renal function, which can be determined by measuring urinary albumin, blood creatinine, and glomerular filtration rate from the time of diagnosis $(4,5)$. Previous clinical trials have shown that close monitoring and controlling of glucose levels and blood pressure can aid in delaying the progression of $\mathrm{DKD}$, but some clinical needs remain a challenge.

Arterial stiffness is often a consequence of the physiological aging process or atherosclerosis, and it has been also described as a biomarker of hypertension (6), subclinical (7) or overt cardiovascular disease, stroke (8), and mortality (9) in the general population. Arterial stiffness is best characterized by measuring the pulse wave velocity (PWV), which is a commonly used biomarker (10-13). Previous studies have found that PWV can predict CKD progression and mortality $(14,15)$. Additionally, the level of PWV is significantly higher in people with diabetes compared to those without $(16,17)$. However, few studies have explored the possible causal relationship between PWV and the progression of diabetic kidney disease in patients with T2D.

To address this research gap, we investigated the relationship between arterial stiffness and renal dysfunction in T2D patients, as well as whether brachial-ankle pulse wave velocity (baPWV) can be used clinically as a predictor of the progression of renal disease.

\section{SUBJECTS, MATERIALS AND METHODS}

\section{Study Population}

This was a retrospective cohort study, in which data were collected from the electronic medical records of T2D patients who underwent baPWV testing in 2015 and were subsequently assessed for the progression of renal disease from 2016-2019 at Yeungnam University Hospital. In total, 391 patients initially qualified for this study. Patients were excluded for any of the following reasons: not aged $21-79$ years $(n=7)$; missing initial estimated glomerular filtration rate data (eGFR; $n=51$ ); chronic kidney disease stage $<3 \mathrm{~b}\left(\mathrm{eGFR} \leq 45 \mathrm{ml} / \mathrm{min} / 1.73 \mathrm{~m}^{2}\right)$; treated with renal replacement therapy at baseline $(n=9)$; presence of peripheral arterial stenosis confirmed by an ankle brachial index (ABI) of $<0.9(n=5)(18,19)$; or follow-up of renal function was not possible $(n=133)$. In total, 186 patients were finally determined to be eligible. The study protocol was developed in accordance with the tenets of the Declaration of Helsinki and reviewed and approved by the institutional review board of the Yeungnam University Hospital (IRB no. 2020-05-020).

\section{Anthropometrics and Laboratory Measurements}

Body mass index (BMI) was calculated as weight divided by height squared $\left(\mathrm{kg} / \mathrm{m}^{2}\right)$. Data on diabetes duration, comorbidities (hypertension, stroke, and coronary artery disease), and use of renin-angiotensin system (RAS) inhibitors were collected. All laboratory tests were performed in the central laboratory of the Yeungnam University Hospital. Venous blood sampling was taken from the antecubital vein after an overnight fast. The levels of serum glucose, glycated hemoglobin (HbAlc),
TABLE 1 | Baseline characteristics according to the presence of peripheral arteria stiffness (baPWV $\geq 1800 \mathrm{~cm} / \mathrm{s}$ ).

\begin{tabular}{|c|c|c|c|}
\hline & $\begin{array}{l}\text { baPWV }<1800 \\
\mathrm{~cm} / \mathrm{s}(n=142)\end{array}$ & $\begin{array}{c}\text { baPWV } \geq 1800 \\
\mathrm{~cm} / \mathrm{s}(n=44)\end{array}$ & $p$ value \\
\hline Age (yrs) & $57.4 \pm 10.2$ & $64.3 \pm 9.5$ & $<0.001$ \\
\hline Male, $n(\%)$ & 95 (66.9\%) & $23(52.3 \%)$ & 0.078 \\
\hline Diabetes duration (yrs) & $8.1 \pm 7.3$ & $12.2 \pm 8.5$ & 0.002 \\
\hline BMI $\left(\mathrm{kg} / \mathrm{m}^{2}\right)$ & $24.8 \pm 5.0$ & $24.1 \pm 3.2$ & 0.258 \\
\hline Hypertension $n(\%)$ & $76(71.0 \%)$ & $31(70.5 \%)$ & 0.091 \\
\hline Stroke $n(\%)$ & $10(7.0 \%)$ & $2(4.5 \%)$ & 0.556 \\
\hline Coronary artery disease $n(\%)$ & $5(3.5 \%)$ & $3(7.0 \%)$ & 0.329 \\
\hline RAS inhibitors, $n(\%)$ & $65(45.8 \%)$ & 27 (61.4\%) & 0.071 \\
\hline Systolic BP (mmHg) & $125.8 \pm 15.4$ & $145.3 \pm 19.1$ & $<0.001$ \\
\hline Diastolic BP (mmHg) & $77.0 \pm 10.2$ & $83.7 \pm 10.8$ & $<0.001$ \\
\hline baPWV (cm/s) & $1490.1 \pm 194.0$ & $2118.6 \pm 308.2$ & $<0.001$ \\
\hline $\mathrm{ABI}$ & $1.15 \pm 0.08$ & $1.17 \pm 0.09$ & 0.020 \\
\hline HbA1c (\%) & $8.3 \pm 2.3$ & $8.6 \pm 1.7$ & 0.230 \\
\hline Total protein (g/dl) & $7.3 \pm 0.7$ & $7.4 \pm 0.5$ & 0.781 \\
\hline Albumin (g/dl) & $4.7 \pm 0.6$ & $4.7 \pm 0.4$ & 0.835 \\
\hline AST (unit/L) & $28.7 \pm 17.5$ & $28.0 \pm 10.1$ & 0.899 \\
\hline ALT (unit/L) & $31.6 \pm 21.9$ & $28.2 \pm 15.4$ & 0.300 \\
\hline Total cholesterol (mg/dl) & $186.1 \pm 59.9$ & $188.1 \pm 50.7$ & 0.634 \\
\hline Triglyceride (mg/dl) & $194.4 \pm 181.9$ & $175.2 \pm 110.6$ & 0.520 \\
\hline HDL cholesterol (mg/dl) & $51.1 \pm 15.5$ & $50.6 \pm 13.3$ & 0.822 \\
\hline LDL cholesterol (mg/dl) & $98.6 \pm 48.5$ & $101.3 \pm 42.4$ & 0.510 \\
\hline Creatinine (mg/dl) & $1.01 \pm 0.20$ & $1.02 \pm 0.22$ & 0.603 \\
\hline eGFR $\left(\mathrm{ml} / \mathrm{min} / 1.73 \mathrm{~m}^{2}\right)$ & $80.8 \pm 16.4$ & $72.6 \pm 14.3$ & 0.005 \\
\hline uACR (mg/g) & $55.7 \pm 163.1$ & $92.2 \pm 172.6$ & 0.172 \\
\hline
\end{tabular}

$B M I$, body mass index; RAS, renin-angiotensin system; BP, blood pressure; baPWV, brachial-ankle pulse wave velocity; $A B I$, ankle brachial index; $A S T$, aspartate aminotransferase; $A L T$, alanine aminotransferase; $H D L$, high-density lipoprotein; $L D L$, low-density lipoprotein; eGFR, estimated glomerular filtration rate; UACR, urinary albumin:creatinine ratio. The bold values mean statistically significant data.

TABLE 2 | Correlation between baPWV and multiple variables at baseline.

\begin{tabular}{lrr}
\hline & \multicolumn{2}{c}{ baPWV } \\
\cline { 2 - 3 } & \multicolumn{1}{c}{$\boldsymbol{r}$} & $\boldsymbol{P}$-value \\
\hline Age & 0.405 & $<0.001$ \\
Diabetes duration & 0.213 & 0.004 \\
Systolic blood pressure & 0.645 & $<0.001$ \\
eGFR & -0.193 & 0.011 \\
\hline
\end{tabular}

total protein, albumin, aspartate aminotransferase (AST), alanine aminotransferase (ALT), total cholesterol, triglyceride, highdensity lipoprotein (HDL) cholesterol, low-density lipoprotein (LDL) cholesterol, blood urea nitrogen (BUN), and creatinine were measured. The levels of urine creatinine and albumin were also measured.

\section{Measurement and Definition of Arterial Stiffness}

The blood pressure (BP), baPWV, and ABI were measured using a non-invasive vascular screening device (BP-203RPEIII, OMRON 
A

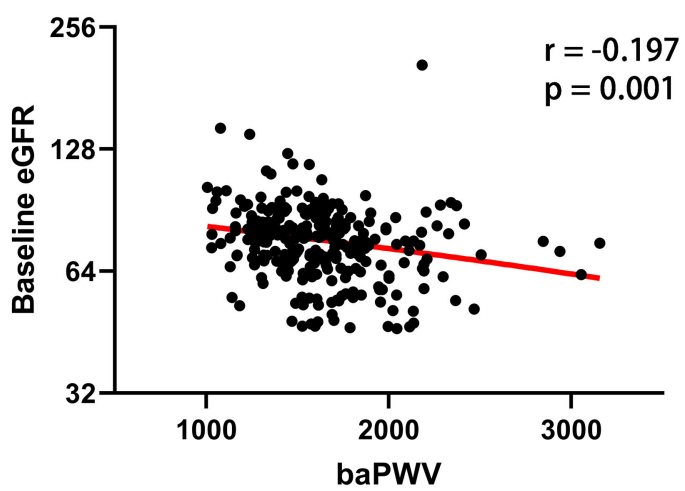

B

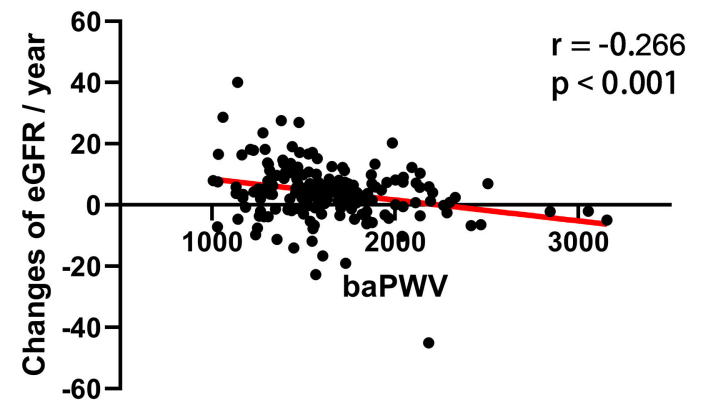

FIGURE 1 | Scatter plot of the relationship between baPWV and (A) baseline eGFR and (B) annual changes in eGFR. The red line represents the regression line.

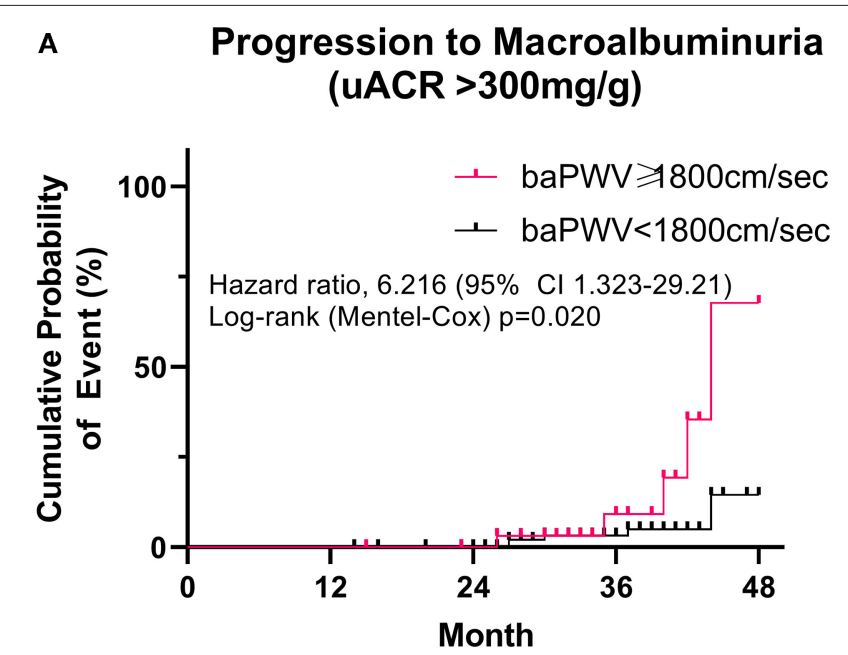

B

Progression of Renal Diseae

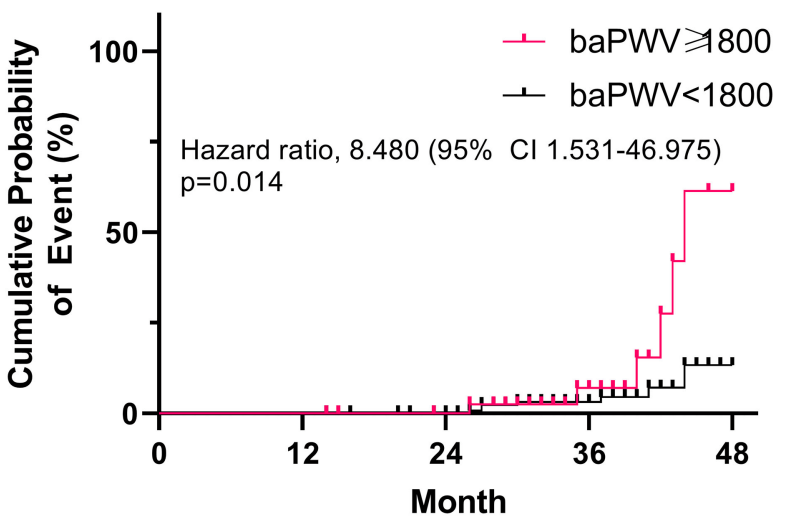

FIGURE 2 | Risk of peripheral arterial stiffness (baPWV $\geq 1800 \mathrm{~cm} / \mathrm{s}$ ) on (A) progression to macroalbuminuria (uACR > $300 \mathrm{mg}$ ) and (B) progression of renal disease. A log-rank (Mantel-Cox) survival analysis was used.

healthcare, Japan), which was operated by a trained examiner who placed pneumatic cuffs on each ankle and each upper arm of patients in the supine position. The validity of baPWV compared to aortic PWV using invasive catheter manometer was reported to be favorable $(r=0.87, p<0.01)(20)$. We defined a person with a baPWV of $\geq 1800 \mathrm{~cm} / \mathrm{s}$ as having a higher level of arterial stiffness, in accordance with the Japanese Circulation Society. A baPWV of $\geq 1800 \mathrm{~cm} / \mathrm{s}$ is regarded as the threshold for a high-risk category (18) and is known to increase the risk for cardiovascular and heart failure-related events $(7,21)$.

\section{Measurement and Definition of the Progression of Renal Disease}

Estimated glomerular filtration rate (eGFR) was calculated using the chronic kidney disease epidemiology collaboration equation (CKD-EPI). Urinary albumin:creatinine ratio (uACR) was calculated. In accordance with the EMPA-REG OUTCOME trial, any of the following criteria were used to describe progression of renal disease (22): progression to macroalbuminuria (uACR > $300 \mathrm{mg}$ ); doubling of serum creatinine level accompanied by an eGFR of $\leq 45 \mathrm{ml} / \mathrm{min} / 1.73 \mathrm{~m}^{2}$; treatment with renal replacement therapy; or death from renal disease.

\section{Statistical Analysis}

Numerical data are expressed as the mean \pm standard deviation (SD), and categorical data are expressed as numbers and percentages. The independent $t$-test was used to compare the groups with continuous variables. The chi-square test was used to compare categorical variables. A Cox proportional hazards regression was used to evaluate the risk of progression of renal disease. IBM Statistical Package for Social Sciences for Windows, version 21.0 (SPSS Inc. Chicago, IL) software was used for statistical analyses. Graphs were produced using GraphPad Prism 8.0 software (GraphPad Software Inc., San Diego, CA, USA). A value of $p<0.05$ was considered statistically significant. 


\section{RESULTS}

\section{Comparison of Baseline Characteristics Between High and Low Pulse Wave Velocity}

The mean age of patients was $59.1 \pm 10.5$ years (range 22-79) and the male:female ratio was $1.73: 1$. The mean duration of diabetes was $9 \pm 7.8$ years (range $0-39$ ). The prevalence of comorbid hypertension, stroke, and coronary artery disease in the patient cohort was $57.5,6.5$, and $4.3 \%$, respectively. $23.7 \%$ of enrolled patients $(n=44)$ had a baPWV of $\geq 1800 \mathrm{~cm} / \mathrm{s}$.

Table 1 lists baseline characteristics according to baPWV ( $\geq$ $1800 \mathrm{~cm} / \mathrm{s}$ vs. $<1800 \mathrm{~cm} / \mathrm{s})$. Individuals with a baPWV of $\geq 1800$ $\mathrm{cm} / \mathrm{s}$ were significantly older $(p<0.001)$, had longer duration of diabetes $(p=0.002)$, higher systolic and diastolic blood pressure $(p<0.01)$, and higher ABI $(p=0.02)$ compared to patients with a baPWV of $<1800 \mathrm{~cm} / \mathrm{s}$. Factors including sex, BMI, comorbid hypertension, stroke or coronary artery disease, and use of RAS inhibitor medication did not differ significantly between the two groups. Baseline HbA1c, albumin, liver and lipid profiles also did

TABLE 3 | Characteristics according to progressors and non-progressors regarding albuminuria.

\begin{tabular}{lccc}
\hline & $\begin{array}{c}\text { Non-progressors } \\
(\boldsymbol{n}=\mathbf{1 7 6})\end{array}$ & $\begin{array}{c}\text { Progressors } \\
(\boldsymbol{n}=\mathbf{1 0})\end{array}$ & $\boldsymbol{p}$ value \\
\hline Age $(\mathrm{yrs})$ & $58.9 \pm 10.5$ & $61.5 \pm 10.6$ & 0.401 \\
Male, $n(\%)$ & $110(62.5 \%)$ & $8(80.0 \%)$ & 0.330 \\
Diabetes duration (yrs) & $8.8 \pm 7.9$ & $12.8 \pm 5.5$ & 0.051 \\
RAS inhibitors, $n(\%)$ & $84(47.7 \%)$ & $8(80.0 \%)$ & 0.056 \\
Systolic BP $(\mathrm{mmHg})$ & $130.4 \pm 18.6$ & $131.2 \pm 11.4$ & 0.623 \\
Diastolic BP $(\mathrm{mmHg})$ & $78.7 \pm 10.8$ & $75.1 \pm 9.1$ & 0.425 \\
baPWV (cm/s) & $1627.8 \pm 348.5$ & $1831.2 \pm 334.8$ & $\mathbf{0 . 0 4 1}$ \\
ABI & $1.16 \pm 0.08$ & $1.17 \pm 0.11$ & 0.913 \\
HbA1c $(\%)$ & $8.3 \pm 2.1$ & $9.0 \pm 2.2$ & 0.199 \\
eGFR $\left(\mathrm{ml} / \mathrm{min} / 1.73 m^{2}\right)$ & $75.9 \pm 18.3$ & $77.7 \pm 12.4$ & 0.465 \\
\hline
\end{tabular}

Mann-Whitney $U$ test, Fisher's exact test was used. The bold values mean statistically significant data. not differ significantly between the two groups. In the baPWV of $\geq 1800 \mathrm{~cm} / \mathrm{s}$ group, eGFR was significantly lower (72.6 vs. 80.8 $\left.\mathrm{ml} / \mathrm{min} / 1.73 \mathrm{~m}^{2}, p=0.005\right)$ and uACR was higher but without statistical significance $(92.2$ vs. $55.7 \mathrm{mg} / \mathrm{g}, p=0.172)$. Table 2 lists correlations between baPWV and age, diabetes duration, systolic blood pressure, and eGFR at baseline. Age, diabetes duration, and systolic blood pressure were positively correlated with baPWV ( $r=0.405,0.213$, and 0.645 , respectively; all $p<0.01)$, whereas baPWV was negatively correlated with eGFR $(r=-0.193, p=$ 0.011; Figure 1A).

\section{Comparison of the Risk for the Progression of Renal Disease Between High and Low Pulse Wave Velocity}

The mean follow-up period was $35.3 \pm 7.25$ months (range 14-48). During the follow-up period, 13 patients developed renal disease $(13.6 \%$ of the baPWV of $\geq 1800 \mathrm{~cm} / \mathrm{s}$ group and $4.9 \%$ of the baPWV of $<1800 \mathrm{~cm} / \mathrm{s}$ group). Specifically, 10 patients progressed to macroalbuminuria and 3 patients exhibited doubling of serum creatinine levels accompanied by an eGFR of $\leq 45 \mathrm{ml} / \mathrm{min} / 1.73 \mathrm{~m}^{2}$. No patients were treated with renal replacement therapy or died from renal disease. The annual changes in eGFR were significantly negatively correlated with baPWV ( $r=-0.226, p<0.001$; Figure 1B), meaning that the higher the baPWV, the greater the annual decrement of eGFR. Individuals with a baPWV of $\geq 1800 \mathrm{~cm} / \mathrm{s}$ had a significantly higher risk for progression to macroalbuminuria (HR 6.216, 95\% CI 1.323-29.21, $p=0.020$; Figure 2A), whereas no significant risk was observed for the doubling of serum creatinine level accompanied by an eGFR of $\leq 45 \mathrm{ml} / \mathrm{min} / 1.73 \mathrm{~m}^{2}$ (HR $2.762,95 \%$ CI $0.146-52.07, p=0.497$ ). The additional analysis of comparing the progressors and non-progressors regarding macroalbuminuria group are presented in Table 3. Compared to the non-progressor group, baseline baPWV was significantly higher in the progressor group (1627.8 vs. $1831.2 \mathrm{~cm} / \mathrm{s}, p=$ 0.041). And although not statistically significant, the duration of diabetes was longer ( 8.8 vs. 12.8 years, $p=0.051$ ) and the rate of taking RAS inhibitors were higher ( 47.7 vs. $80.0 \%, p=0.056)$ in the progressor group. Table 4 lists the risk of arterial stiffness for

TABLE 4 | Risk of peripheral arterial stiffness (baPWV $\geq 1800 \mathrm{~cm} / \mathrm{s}$ ) on the progression of renal disease.

\begin{tabular}{|c|c|c|c|c|c|c|}
\hline & \multicolumn{3}{|c|}{ Non-adjusted model } & \multicolumn{3}{|c|}{ Adjusted model* } \\
\hline & HR & $95 \% \mathrm{Cl}$ & $p$-value & HR & $95 \% \mathrm{Cl}$ & $p$-value \\
\hline Age & 1.008 & [0.954-1.064] & 1.008 & 0.965 & [0.898-1.037] & 0.330 \\
\hline Male & 3.078 & [0.681-13.913] & 0.144 & 5.009 & [0.838-29.952] & 0.077 \\
\hline Systolic BP & 0.986 & [0.953-1.021] & 0.443 & 0.993 & [0.923-1.068] & 0.847 \\
\hline Diastolic BP & 0.962 & [0.909-1.019] & 0.180 & 0.926 & {$[0.823-1.041]$} & 0.197 \\
\hline Diabetes duration & 1.038 & [0.983-1.095] & 0.185 & 1.017 & [0.945-1.094] & 0.653 \\
\hline Baseline eGFR & 0.977 & [0.959-0.995] & 0.015 & 1.003 & [0.986-1.020] & 0.741 \\
\hline RAS inhibitor medication & 2.598 & [0.789-8.559] & 0.116 & 2.097 & {$[0.576-7.631]$} & 0.261 \\
\hline baPWV $\geq 1800 \mathrm{~cm} / \mathrm{s}$ & 3.271 & [1.096-9.769] & 0.034 & 8.480 & {$[1.531-46.975]$} & 0.014 \\
\hline
\end{tabular}

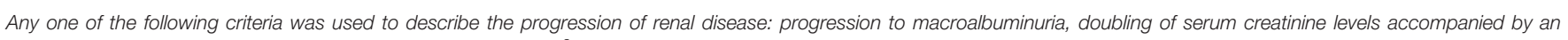
estimated glomerular filtration rate (eGFR) of $\leq 45 \mathrm{ml} / \mathrm{min} / 1.73 \mathrm{~m}^{2}$, treatment with renal replacement therapy, or death from renal disease.

${ }^{*}$ Adjusted for age, sex, systolic BP, diastolic BP, diabetes duration, baseline eGFR, use of RAS inhibitors, and baPWV. The bold values mean statistically significant data. 
progression of renal disease based on a Cox-regression analysis. Age, sex, systolic BP, diastolic BP, diabetes duration, eGFR, use of RAS inhibitors, and baPWV were considered as covariates. In non-adjusted regression analysis, the lower baseline eGFR, and baPWV of $\geq 1800 \mathrm{~cm} / \mathrm{s}$ increased the risk of progression of renal disease. After adjustments, individuals with a baPWV of $\geq$ $1800 \mathrm{~cm} / \mathrm{s}$ had an increased risk for progression of renal disease compared to those with a baPWV of $<1800 \mathrm{~cm} / \mathrm{s}$ (HR 8.480, 95\% CI 1.531-46.975, $p=0.014$; Figure 2B).

\section{DISCUSSION}

After following patients for an average duration of 35 months, we found that T2D patients with a baPWV $\geq 1800 \mathrm{~cm} / \mathrm{s}$ are at 8.5-fold greater risk of progression of renal disease, regardless of classical risk factors. This finding suggests that peripheral arterial stiffness represented by a high baPWV may accelerate the progression of CKD in patients with type 2 diabetes.

Many previous studies have suggested that arterial (or aortic) stiffness is closely related with the risk of mortality and specifically cardiovascular disease, not only in the general population but also in patients with underlying comorbidities such as hypertension and diabetes $(6,8-10)$. The CRIC study (Chronic Renal Insufficiency Cohort) revealed that high aortic stiffness increases the risk of chronic kidney disease progression and all-cause death in patients with impaired kidney function (23). Other studies have demonstrated that arterial stiffness is increased in patients with T2D (24) and that PWV can be used as a predictor for cardiovascular events and all-cause mortality (17). Arterial stiffness may cause left ventricular hypertrophy and dysfunction due to higher pressure pulse pulsation (19). A plausible explanation for the impact of arterial stiffness on the progression of renal disease is that arterial stiffness increases hemodynamic shear stress, which may result in endothelial dysfunction and microvascular ischemia, ultimately causing kidney injury $(25,26)$.

High PWV has been shown to be associated with diabetic retinopathy (27), neuropathy $(28,29)$, and nephropathy. A Rotterdam study of 3,666 subjects from the general population included an 11-year follow-up and revealed that carotidfemoral pulse wave velocity (cfPWV) was associated with a rapid reduction in eGFR and CKD progression (14). Another 9-year follow-up of $211 \mathrm{~T} 2 \mathrm{D}$ patients in the UK revealed that cfPWV was associated with a decrease in eGFR among patients under 60 years of age (30). A study in Singapore involving 1012 T2D patients of differing Asian ethnicities and an average follow-up period of 3.1 years revealed that cfPWV was an independent predictor for albuminuria progression (31). These results indicate that $\mathrm{cfPWV}$ can be a predictor of DKD progression in T2D. Nevertheless, a study involving 913 subjects from the general Korean population and an average follow-up period of 3.2 years found that neither cfPWV nor baPWV are related to renal dysfunction and that the significance of baPWV in T2D patients is likely invalid due to the relatively small proportion of T2D patients (32). The most important aspect of the present study is that baPWV was associated with a decrease in renal function when Korean patients with T2D were followed-up for an average of 3 years.
Additionally, the present study used the validated cut-off value for baPWV and demonstrated that this association exists, despite applying very strict criteria for the progression of renal disease.

A study of 461 Japanese T2D patients, with an average of 5.9 years follow-up, investigated the risks of arterial stiffness on progression of renal disease in T2D. It found that patients with a cfPWV of $\geq 910 \mathrm{~cm} / \mathrm{s}$ were at increased risk of albuminuria (transition from normo- to micro-albuminuria or from micro- to macro-albuminuria; $\mathrm{HR}=1.23$ ), and a linear regression analysis revealed a negative correlation between cfPWV and annual change in eGFR (33). Our study produced similar results: patients with a baPWV of $\geq 1800 \mathrm{~cm} / \mathrm{s}$ were at increased risk of progression of renal disease; specifically, progression to macroalbuminuria and the annual change of eGFR were negatively correlated with baPWV. In addition to cfPWV, baPWV would be a useful screening tool for predicting progression of renal disease in $\mathrm{T} 2 \mathrm{D}$ patients, due to the ease and convenience of measurements.

This study had a limitation that it enrolled a relatively small number of patients and used the single measurement of baPWV. However, to our knowledge, this is the first study to identify the risk of peripheral arterial stiffness on the progression of renal disease with the capability of distinguishing a causal relationship.

In conclusion, baPWV representing peripheral arterial stiffness can be used as a predictor of the progression of renal disease in T2D patients.

\section{DATA AVAILABILITY STATEMENT}

The raw data supporting the conclusions of this article will be made available by the authors, without undue reservation.

\section{ETHICS STATEMENT}

The studies involving human participants were reviewed and approved by Institutional review board of the Yeungnam University Hospital. The patients/participants provided their written informed consent to participate in this study.

\section{AUTHOR CONTRIBUTIONS}

SMC and JSM: conceptualization and study design. THL, DSL, and SRC: data collection. THL, SMC, DSL, and SRC: data analysis and interpretation. THL, SMC, and JSM: writing. JSY, KCW, and HWL: review and editing. All authors contributed to the article and approved the submitted version.

\section{FUNDING}

The present study was supported by a National Research Foundation of Korea grant funded by the Korean government (grant no. NRF-2020R1A2C4002626, NRF2019M3E5D1A02068104). The funders had no role in study design, data collection and analysis, decision to publish or preparation of the manuscript. 


\section{REFERENCES}

1. Won JC, Lee JH, Kim JH, Kang ES, Won KC, Kim DJ, et al. Diabetes fact sheet in Korea, 2016: an appraisal of current status. Diabetes Metab J. (2018) 42:415-24. doi: 10.4093/dmj.2018.0017

2. ESRD Registry Committee: Korean Society of Nephrology. Current Renal Replacement Therapy in Korea. (2019). Available online at: http://www.ksn. or.kr/rang_board/list.html?code=sinchart_eng

3. Kim SH, Jo MW, Go DS, Ryu DR, Park J. Economic burden of chronic kidney disease in Korea using national sample cohort. J Nephrol. (2017) 30:787-93. doi: 10.1007/s40620-017-0380-3

4. National Kidney F. KDOQI clinical practice guideline for diabetes and CKD: 2012 update. Am J Kidney Dis. (2012) 60:850-86. doi: 10.1053/j.ajkd.2012.07.005

5. American Diabetes Association. Microvascular Complications and Foot Care: Standards of Medical Care in Diabetes-2020. Diabetes Care. (2020). 43(Suppl 1):S135-51. doi: $10.2337 / \mathrm{dc} 20-S 011$

6. Oh YS. Arterial stiffness and hypertension. Clin Hypertens. (2018) 24:17. doi: 10.1186/s40885-018-0102-8

7. Chung YK, Lee YJ, Kim KW, Cho RK, Chung SM, Moon JS, et al. Serum cystatin $\mathrm{C}$ is associated with subclinical atherosclerosis in patients with type 2 diabetes: A retrospective study. Diab Vasc Dis Res. (2018) 15:2430. doi: $10.1177 / 1479164117738156$

8. Mattace-Raso FU, van der Cammen TJ, Hofman A, van Popele NM, Bos ML, Schalekamp MA, et al. Arterial stiffness and risk of coronary heart disease and stroke: the Rotterdam Study. Circulation. (2006) 113:65763. doi: 10.1161/CIRCULATIONAHA.105.555235

9. Cruickshank K, Riste L, Anderson SG, Wright JS, Dunn G, Gosling RG. Aortic pulse-wave velocity and its relationship to mortality in diabetes and glucose intolerance: an integrated index of vascular function? Circulation. (2002) 106:2085-90. doi: 10.1161/01.CIR.0000033824.02722.F7

10. Yamashina A, Tomiyama H, Arai T, Hirose K, Koji Y, Hirayama Y, et al. Brachial-ankle pulse wave velocity as a marker of atherosclerotic vascular damage and cardiovascular risk. Hypertens Res. (2003) 26:61522. doi: 10.1291/hypres.26.615

11. Covic A, Haydar AA, Bhamra-Ariza P, Gusbeth-Tatomir P, Goldsmith DJ. Aortic pulse wave velocity and arterial wave reflections predict the extent and severity of coronary artery disease in chronic kidney disease patients. $J$ Nephrol. (2005) 18:388-96.

12. Munakata M, Sakuraba J, Tayama J, Furuta T, Yusa A, Nunokawa T, et al. Higher brachial-ankle pulse wave velocity is associated with more advanced carotid atherosclerosis in end-stage renal disease. Hypertens Res. (2005) 28:914. doi: 10.1291/hypres.28.9

13. Avolio A. Arterial stiffness. Pulse (Basel). (2013) 1:1428. doi: $10.1159 / 000348620$

14. Sedaghat S, Mattace-Raso FU, Hoorn EJ, Uitterlinden AG, Hofman A, Ikram MA, et al. Arterial Stiffness And Decline In Kidney Function. Clin J Am Soc Nephrol. (2015) 10:2190-7. doi: 10.2215/CJN.03000315

15. van Varik BJ, Vossen LM, Rennenberg RJ, Stoffers HE, Kessels AG, de Leeuw PW, et al. Arterial stiffness and decline of renal function in a primary care population. Hypertens Res. (2017) 40:73-8. doi: 10.1038/hr.2016.113

16. Taniwaki H, Kawagishi T, Emoto M, Shoji T, Kanda H, Maekawa K, et al. Correlation between the intima-media thickness of the carotid artery and aortic pulse-wave velocity in patients with type 2 diabetes. Vessel wall properties in type 2 diabetes. Diabetes Care. (1999) 22:18517. doi: $10.2337 /$ diacare.22.11.1851

17. Cardoso CR, Ferreira MT, Leite NC, Salles GF. Prognostic impact of aortic stiffness in high-risk type 2 diabetic patients: the Rio deJaneiro Type 2 Diabetes Cohort Study. Diabetes Care. (2013) 36:3772-8. doi: 10.2337/ dc13-0506

18. Munakata M. Brachial-ankle pulse wave velocity in the measurement of arterial stiffness: recent evidence and clinical applications. Curr Hypertens Rev. (2014) 10:49-57. doi: 10.2174/15734021100114111 1160957
19. Kim HL, Kim SH. Pulse wave velocity in atherosclerosis. Front Cardiovasc Med. (2019) 6:41. doi: 10.3389/fcvm.2019.00041

20. Yamashina A, Tomiyama H, Takeda K, Tsuda H, Arai T, Hirose K, et al. Validity, reproducibility, and clinical significance of noninvasive brachialankle pulse wave velocity measurement. Hypertens Res. (2002) 25:35964. doi: 10.1291/hypres.25.359

21. Takae M, Yamamoto E, Tokitsu T, Oike F, Nishihara T, Fujisue K, et al. Clinical significance of brachial-ankle pulse wave velocity in patients with heart failure with reduced left ventricular ejection fraction. Am J Hypertens. (2019) 32:657-67. doi: 10.1093/ajh/hpz048

22. Wanner C, Inzucchi SE, Lachin JM, Fitchett D, von Eynatten M, Mattheus M, et al. Empagliflozin and progression of kidney disease in type 2 diabetes. $N$ Engl J Med. (2016) 375:323-34. doi: 10.1056/NEJMoa1515920

23. Townsend RR, Anderson AH, Chirinos JA, Feldman HI, Grunwald JE, Nessel L, et al. Association of pulse wave velocity with chronic kidney disease progression and mortality: findings from the CRIC Study (Chronic Renal Insufficiency Cohort). Hypertension. (2018) 71:11017. doi: 10.1161/HYPERTENSIONAHA.117.10648

24. Schram MT, Henry RM, van Dijk RA, Kostense PJ, Dekker JM, Nijpels $\mathrm{G}$, et al. Increased central artery stiffness in impaired glucose metabolism and type 2 diabetes: the Hoorn Study. Hypertension. (2004) 43:17681. doi: 10.1161/01.HYP.0000111829.46090.92

25. Chung SM, Oh JH, Moon JS, Kim YK, Yoon JS, Won KC, et al. Critical shear stress is associated with diabetic kidney disease in patients with type 2 diabetes. Sci Rep. (2018) 8:908. doi: 10.1038/s41598-018-25252-8

26. Safar ME, London GM, Plante GE. Arterial stiffness and kidney function. Hypertension. (2004) 43:163-8. doi: 10.1161/01.HYP.0000114571.75762.b0

27. Yun YW, Shin MH, Lee YH, Rhee JA, Choi JS. Arterial stiffness is associated with diabetic retinopathy in Korean type 2 diabetic patients. J Prev Med Public Health. (2011) 44:260-6. doi: 10.3961/jpmph.2011.44.6.260

28. Ha BK, Kim BG, Kim DH, Lee SI, Jung SM, Park JY, et al. Relationships between brachial-ankle pulse wave velocity and peripheral neuropathy in type 2 diabetes. Diabetes Metab J. (2012) 36:443-51. doi: 10.4093/dmj.2012.36.6.443

29. Wu N, Cai X, Ye K, Li Y, He M, Zhao W, et al. Association between BrachialAnkle pulse wave velocity and cardiac autonomic neuropathy in type 2 diabetes. Diabetol Metab Syndr. (2014) 6:82. doi: 10.1186/1758-5996-6-82

30. Fountoulakis N, Thakrar C, Patel K, Viberti G, Gnudi L, Karalliedde J. Increased arterial stiffness is an independent predictor of renal function decline in patients with type 2 diabetes mellitus younger than 60 years. $J \mathrm{Am}$ Heart Assoc. (2017) 6:4. doi: 10.1161/JAHA.116.004934

31. Zhang X, Low S, Sum CF, Tavintharan S, Yeoh LY, Liu J, et al. Arterial stiffness is an independent predictor for albuminuria progression among Asians with type 2 diabetes-A prospective cohort study. J Diabetes Complications. (2017) 31:933-8. doi: 10.1016/j.jdiacomp.2017.02.004

32. Kim CS, Kim HY, Kang YU, Choi JS, Bae EH, Ma SK, et al. Association of pulse wave velocity and pulse pressure with decline in kidney function. J Clin Hypertens (Greenwich). (2014) 16:372-7. doi: 10.1111/jch.12302

33. Bouchi R, Babazono T, Mugishima M, Yoshida N, Nyumura I, Toya K, et al. Arterial stiffness is associated with incident albuminuria and decreased glomerular filtration rate in type 2 diabetic patients. Diabetes Care. (2011) 34:2570-5. doi: $10.2337 / \mathrm{dc} 11-1020$

Conflict of Interest: The authors declare that the research was conducted in the absence of any commercial or financial relationships that could be construed as a potential conflict of interest.

Copyright (c) 2020 Lim, Chung, Lee, Choi, Moon, Yoon, Won and Lee. This is an open-access article distributed under the terms of the Creative Commons Attribution License (CC BY). The use, distribution or reproduction in other forums is permitted, provided the original author(s) and the copyright owner(s) are credited and that the original publication in this journal is cited, in accordance with accepted academic practice. No use, distribution or reproduction is permitted which does not comply with these terms. 